\title{
Multichannel near-field nanoscopy of circular and linear dichroism at the solid-state
}

\author{
G. Lazzini ${ }^{1}$, M. Castriciano ${ }^{2}$, M. Trapani ${ }^{2}$, N. Micali ${ }^{3}$, F. Tantussi ${ }^{1}$,

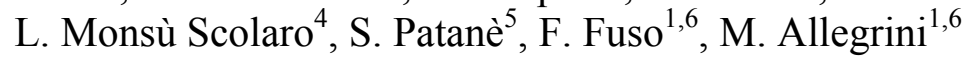 \\ ${ }^{1}$ Dipartimento di Fisica "Enrico Fermi” and CNISM, Università di Pisa, 56127 Pisa, Italy \\ ${ }^{2}$ CNR-ISMN, Istituto per lo Studio dei Materiali Nanostrutturati, 98166 Messina, Italy \\ ${ }^{3}$ CNR-IPCF, Istituto per i Processi Chimico-Fisici, 98166 Messina, Italy \\ ${ }^{4}$ Dipartimento di Scienze Chimiche, Biologiche, Farmaceutiche, Ambientali, \\ Università di Messina, 98166 Messina, Italy \\ ${ }^{5}$ Dipartimento di Scienze matematiche e informatiche, scienze fisiche e scienze della terra, \\ Università di Messina, 98166 Messina, Italy \\ ${ }^{6}$ CNR-INO, Istituto Nazionale di Ottica, 56124 Pisa, Italy
}

\begin{abstract}
We investigate the optical response of individual porphyrin (TPPS 3 ) nanoaggregates anchored onto a glass substrate by using a specific configuration of Polarization-Modulation Scanning Near-Field Optical Microscopy (PM-SNOM). Subdiffraction spatial resolution and sensitivity to the chiroptical properties of the material is reported. By demodulating the transmitted signal at the first and the second harmonics, the response of the nanoggregates to circular and linear polarization is simultaneously acquired in the same scan. In order to evaluate the relevant dichroic coefficients, we analyze a sample comprising several tens of individual nanoaggregates by using a model based on the Mueller matrix formalism. Circular dichroism in the nanoaggregates is demonstrated to stem from their molecular structure, whereas linear dichroism occurs as a consequence of the strongly anisotropic shape of the deposited nanoaggregates.
\end{abstract}

Keywords: Nanospectroscopy, Circular dichroism at the solid-state, Linear dichroism at the solid-state, Near-field optics, Polarization-modulation, Nanosensing

\section{INTRODUCTION}

The role of chirality at the molecular level and its expression at the nano- and micro-scales are among the most intriguing topics in molecular biology ${ }^{1}$. Many molecular building blocks of biological entities, such as amino acids and sugars, exhibit chiral structures. The origin of homochirality in most naturally occurring molecules of such classes is the subject of strong debates since several decades.

Chirality is typically associated with Circular Dichroism (CD), i.e., the differential absorption of left- and right-handed circularly polarized light. Since the discovery of the optical rotatory dispersion ascribed to Aimé Cotton ${ }^{2}$, many efforts have been devoted to develop methods and tools suitable for CD investigations. Nowadays, CD spectroscopy is widely used to determine molecular chirality.

CD spectroscopy, however, requires collecting and analyzing absorption spectra of vibrational transitions, which can be effectively accomplished only in the liquid phase, i.e., in spatially disordered ensembles comprising a relevant number of individual absorbers. Such a limitation, which is not relevant for most biochemical investigations involving molecular species typically dissolved in solutions, hampers studying chirality at the solid-state.

We use a variant of Polarization-Modulation Scanning Near-Field Optical Microscopy (PM-SNOM) ${ }^{3}$ to investigate the optical activity of molecular nanoaggregates showing both CD and linear dichroism (LD). Thanks to the sub-diffraction spatial resolution typical of near-field microscopy, individual ribbon-shaped nanoaggregates are addressed. Furthermore, the simultaneous acquisition of topography and optical maps enables the identification and morphological characterization of the nanostructures. The strongly confined interaction between the localized near-field and the opticalactive material leads to an excellent sensitivity, adequate for investigating the single nanoaggregate. 
The optical signal resulting from the interaction between the near-field and the nanoaggregates is collected by a detector placed below the sample, hence mimicking the transmission microscopy configuration in conventional optics measurements. Demodulation of the collected signal at the first and the second harmonics with respect to the polarization-modulation allows us to produce multichannel maps of the optical activity, representative of CD and LD, as well as of the polarization-averaged optical transmission ${ }^{4}$. Contrary to ordinary CD spectroscopy, where the response to linear polarization is smeared out due to the random orientation of the molecular absorbers in the investigated ensemble, our approach leads to effectively disentangle the contribution of CD and LD to the optical activity and eventually to the quantitative evaluation of the relevant coefficients.

Aggregates of tris-(4-sulphonatophenyl)phenyl-porphyrin (TPPS 3 ), deposited from the liquid phase onto glass substrates and showing remarkable $\mathrm{CD}$ in solution, are analyzed. The results of our nanoscopy approach are relevant for enlightening the origin of the optical activity in such supra-molecular aggregates. The investigation of CD and LD at the local scale can also provide significant outcomes in the assessment of optically active nanostructures intended for advanced nanophotonics applications, e.g., in active chiral plasmonics ${ }^{5,6}$.

\section{EXPERIMENTAL}

\subsection{PM-SNOM setup}

A customized SNOM setup based on an original multi-purpose head ${ }^{7}$ is employed for these investigations. The SNOM operates in the emission-mode. A commercial near-field probe (LovaLite SM-050), featuring a tapered and Al-coated single-mode optical fiber with a $50 \mathrm{~nm}$ (nominal) apical aperture, plays the role of quasi-point like near-field source. The sample is glued on the scanner, consisting of a hollow piezo-electric (PZT) cylinder capable of a maximum travel $7 \mu \mathrm{m} x$ $7 \mu \mathrm{m} \times 2 \mu \mathrm{m}$, the latter referred to the vertical direction. An achromatic lens $(f=5 \mathrm{~mm}, \mathrm{NA}=0.7)$ and a miniaturized photomultiplier (Hamamatsu R7400-U), hosted within the hollow cylinder, collect and detect the optical signal resulting from the local interaction between the near-field and the investigated nanostructure.

Conventional shear-force method is used to operate in constant gap conditions. To this aim, the end of the optical fiber probe is glued onto one pronge of a quartz tuning fork and the whole system is kept in oscillation parallel to the sample surface, at a frequency close to the mechanical resonance (around $32 \mathrm{kHz}$ ). The oscillation amplitude signal, measured by the tuning fork, is converted into a continuous voltage through heterodyne demodulation, and compared to a reference value in order to realize a feedback loop. The comparator output, duly conditioned, drives the vertical displacement of the piezo-electric scanner. In typical operating conditions, a tip-to-surface distance below $10 \mathrm{~nm}$ is achieved, ensuring that the sample surface is concerned by the near-field.

Solid-state laser light (473 $\mathrm{nm}$ or $532 \mathrm{~nm}$ ) at moderate power (below $2 \mathrm{~mW}$, in order to prevent probe damage) is coupled to the optical fiber probe by using a single-mode fiber launcher (Oz-Optics). Core of the polarizationmodulation setup is a photoelastic modulator (PEM, Hinds Instruments PEM-100) placed before the fiber launcher with its optical axes at 45 degrees with respect to the laser polarization direction.

The modulator acts like a waveplate introducing a periodically variable dephasing $\Delta \phi(t)$ between the mutually orthogonal in-plane components of the electric field:

$$
\Delta \phi(t)=A \cos (2 \pi f t)
$$

with $A$ the maximum retardation of the waveplate, user selectable, and $f=50 \mathrm{kHz}$. As an example, Fig. 1 shows the polarization state of the laser radiation at the PEM output in the case $A=\pi / 2$, calculated at different times $t$ (in units of the modulation period $T=2 \pi / f$ ). In such conditions, circular polarization is achieved twice in a single modulation period, yet with distinct signs, corresponding to left- and right-handed directions. As discussed in the following, this circumstance enables detecting both $\mathrm{CD}$ and LD. In order to prevent variations of the residual birefringence from the optical fiber probe, which could spuriously affect the polarization state, a fiber paddle is used.

The detector output is split into several channels and sent to distinct lock-in amplifiers (Stanford Research Systems SR830DSP). One of them, used to produce a signal representative of the polarization-averaged optical transmission of the sample, hereafter denoted as DC, is referenced with a low frequency $\left(f^{\prime}<<f\right)$ amplitude modulation of the laser light. Other two lock-in amplifiers are referenced with the first and second harmonics, respectively, of the polarization 
modulation, providing the signals hereafter denoted as Ist and IInd, which are representative of CD and LD, respectively. All channels are simultaneously acquired during each scan, typically consisting of $256 \times 256$ acquisition points (pixels). Comparison between maps acquired during the forward and the reverse scans allows us to get rid of the finite integration time of the lock-in amplifiers (typically set to 10-30 ms) and to remove the associated spatial shift. In addition, during every scan a topography map is acquired by recording the signal produced by the shear-force feedback loop.

Figure 2 reports a schematic picture of the whole setup.

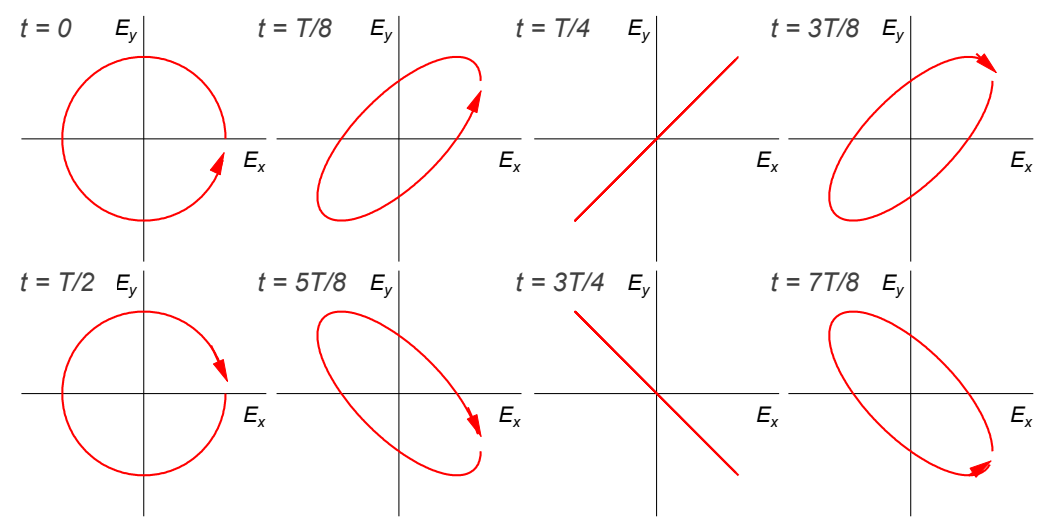

Figure 1. Polarization states of the electric field produced out of the photoelastic modulator at different times $t$, in units of the modulation period $T$. The axes report the field components along two mutually orthogonal in-plane directions, $x$ and $y$. Calculations are made assuming $A=\pi / 2$.

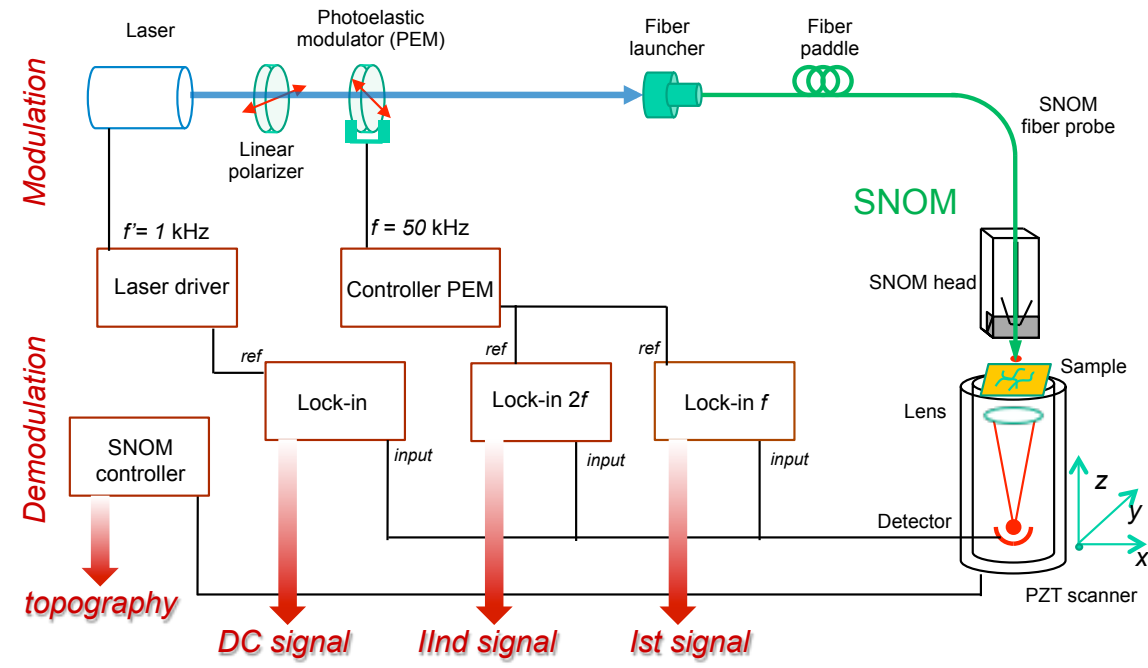

Figure 2. Schematic picture of the whole setup. The available optical channels are named as in the text.

\section{2 $\mathrm{TPPS}_{3}$ sample preparation}

Molecular nanoaggregates of $\mathrm{TPPS}_{3}$ have been prepared from the liquid phase and deposited onto glass slides. Acidification of a $3 \times 10^{-6} \mathrm{M}$ solution of $\mathrm{TPPS}_{3}$ has been achieved with aqueous $\mathrm{HCl} 0.5 \mathrm{M}$. Aggregation has been triggered by adding $0.1 \mathrm{M} \mathrm{NaCl}$ to the solution. The glass substrates have been prepared by cleaning with piranha solution, followed by silanization through immersion in a trimethylchlorosilane solution in $\mathrm{CH}_{2} \mathrm{Cl}_{2}$. The so-prepared substrates have been dipped in the solution of the growing $\mathrm{TPPS}_{3}$ aggregates and left immersed for three days.

In specific hydrodynamic conditions ensuring reversal of the effective gravity experienced by the molecules, it has been shown that the chirality of the supra-molecular porphyrin assemblies can be modified ${ }^{8}$. However, the grown aggregates exhibit symmetry breaking and circular dichroism also in standing solutions 9 . In the liquid phase, the nanoaggregates 
show a rather narrow absorption J-band around $490 \mathrm{~nm}$, red shifted with respect to the corresponding absorption of monomeric porphyrin, accompanied with remarkable signals in the corresponding CD spectra ${ }^{4}$.

\section{RESULTS AND DISCUSSION}

\subsection{Topography and optical transmission}

A non-homogenous distribution of individual, ribbon-shaped, nanoaggregates is found on the substrate. Figure 3 shows as an example the topography (a) and the DC signal (b) maps acquired in the magnified scan over a portion of a single nanoaggregate, carried out upon excitation at $473 \mathrm{~nm}$. Panel (c) in the same figure reports representative cross sections.

The figure clearly illustrates several common features of the investigated nanostructures. First of all, the ribbon shape of the nanoaggregaets is confirmed, being their width much smaller than the height (typically, a few hundreds nm and a few tens $\mathrm{nm}$, respectively). This observation, in agreement with previous Atomic Force Microscopy analyses on similar nanostructures ${ }^{10}$, suggests that, in the early stage of their formation, the J-aggregates organize their structure in the form of nano-helices with defined handedness, which, once deposited onto the solid substrate, give rise to ribbon-shaped nanostructures.

The near-field optical analysis, Fig. 3(b), demonstrates that such nanostructures absorb the electromagnetic field at 473 $\mathrm{nm}$, a wavelength well within the absorption band of the J-aggregates, as ascertained by UV-vis spectroscopy in solution ${ }^{4}$. Therefore, mapping the DC signal allows us to build the spatial distribution of the optical thickness of the nanostructures at the local scale. Remarkably, scans carried out upon excitation at $532 \mathrm{~nm}$, a wavelength out of the absorption band, lead to much smaller dynamics in the DC signal maps dominated by effects related to near-field scattering at the edges of the nanostructures ${ }^{4}$.
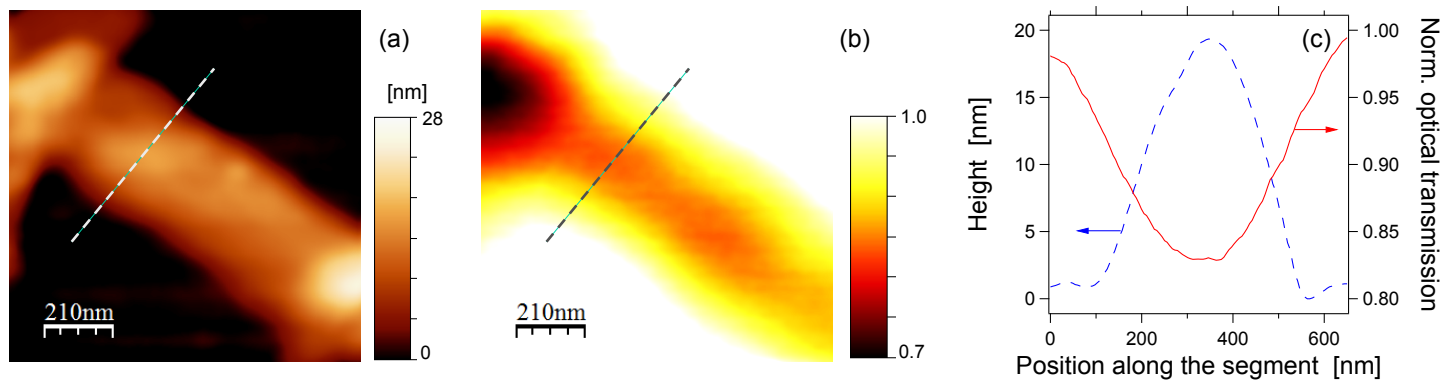

Figure 3. Topography (a) and DC signal, i.e., polarization averaged optical transmission (b), maps showing the magnified view of a single, ribbon-shaped, TPPS $_{3}$ nanoaggregate. The optical transmission is normalized to unity. Panel (c) shows the cross section of both maps (continuous and dashed lines for optical transmission and topography, respectively) along the dashed segment superposed to the maps.

\subsection{Maps of the optical activity at the local scale}

Polarization modulation is a widely adopted strategy to improve the contrast in both conventional and near-field optical microscopy ${ }^{3}$. Despite the non propagating character of the near-field and its complicated spatial distribution, experiments have definitely shown that specifically conceived PM implementations can be used to map the optical activity of materials and devices at the local scale ${ }^{4,11-15}$. Typically, the task requires suitable probes, showing negligible residual birefringence, and the development of a model able to interpret the experimental findings, as will be briefly outlined in Section 3.3. The main result of such a model is that maps of the Ist and IInd signals, i.e., obtained through demodulation at the first and second harmonics, bring distinct quantitative information on $\mathrm{CD}$ and $\mathrm{LD}$, respectively.

Figure 4 shows an example of a scan over a small portion of the sample. A few individual ribbon-shaped nanoaggregates can be identified in the topography map [Fig. 4(a)]. They are oriented along different directions as a consequence of the fabrication process, where no control on the orientation is realized. The corresponding optical maps, shown in panels (b) and (c), obtained through demodulation at the first and the second harmonics, respectively, exhibit a variation of the signal with respect to the average level for most of the identified nanostructures. The average level of the map 
corresponds to regions where no nanostructure is present, hence no optical activity, either linear or circular, is found. Therefore, the observed variations of the signal can be ascribed to the occurrence of dichroism.

Remarkably, while the map obtained through demodulation at the first harmonics (b) shows an almost constant signal, always larger than the average value when measured on the nanostructures, demodulation at the second harmonics (c) reveals signals which can be either larger or smaller than the average value of the map. As confirmed by a comprehensive analysis of several scans carried out on different portions of the sample, such a behavior is a common finding, being observed in over $75 \%$ of the instances. Furthermore, the increase or decrease of the signal with respect to the average value of the map turns to depend on the orientation the nanostructures in the scan plane, i.e. on the angle between their long axis and a reference direction in the map. This is well observed in Fig. 4(c), where the elongated nanostructure directed approximately along the vertical direction of the scan, as well as the portion of nanostructure at its left side, appear dark (according to the used palette) in contrast with other features. The same nanostructures are observed as bright features (according to the used palette) in Fig. 4(b). It is also worth noticing that dark features appear also in Fig. 4(b), but they cannot be ascribed to ribbon-shaped nanoggregates. In fact, the fabrication process does not prevent deposition of unstructured porphyrin from the solution.
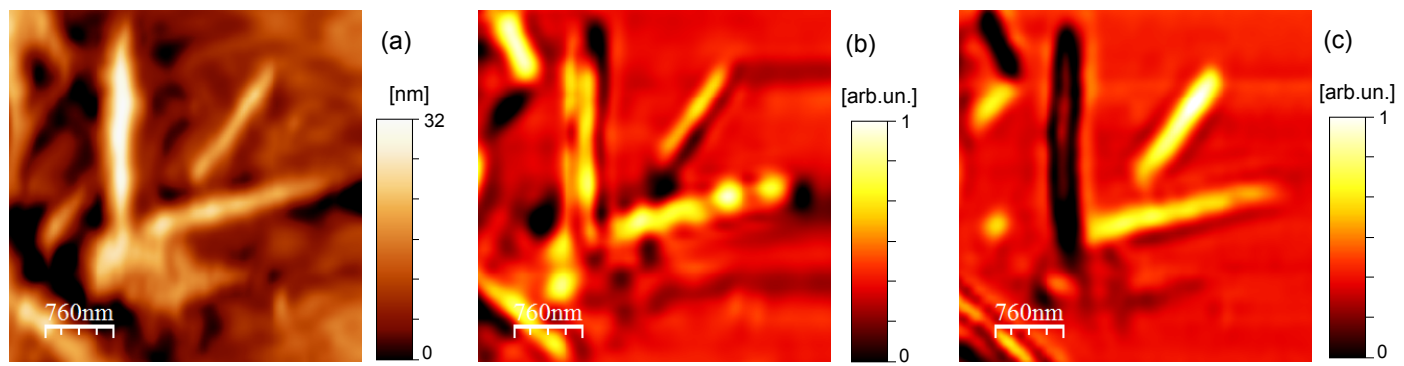

Figure 4. Topography (a), Ist (b) and IInd (c) signal maps acquired during the scan of a portion of the sample showing several distinct nanoaggregates. Scales of the optical maps are expressed in arbitrary units, normalized to unity.

\subsection{Model and data interpretation}

The experiment has been modeled based on the Mueller matrix formalism. In particular, a combination of four distinct $4 \times 4$ matrices is used to take into account of, respectively: (i) the time dependent retardation introduced by the photoelastic modulator; (ii) the spurious birefringence produced by the optical fiber probe; (iii) the LD of the nanostructures, measured by the normalized coefficient $\alpha_{L D}$; (iv) the CD of the nanostructures, measured by the normalized coefficient $\alpha_{C D}$.

Assuming a linear polarization directed along the $x$ direction in the lab reference frame, the radiation at the input of the whole system is expressed by the Stokes vector

$$
\vec{S}_{\text {in }}=I_{0}\left(\begin{array}{c}
1 \\
1 \\
0 \\
0
\end{array}\right)
$$

with $I_{0}$ the incident intensity. The radiation at the output of the whole system, i.e., impinging onto the detector, can be written as

$$
\vec{S}_{\text {out }}(t)=M_{i v}\left(\alpha_{C D}\right) M_{i i i}\left(\alpha_{L D}, \beta\right) M_{i i}\left(\Delta \phi_{s p}\right) M_{i}(t) \vec{S}_{\text {in }}
$$

where $M_{i-i v}$ represent the Mueller matrices accounting for the above mentioned effects. Their explicit form, not reported here for the sake of conciseness, has been evaluated according to the literature, starting from the corresponding Jones matrices for generic birefringent and dichroic components ${ }^{16}$. Such matrices depend on several variables and parameters, as explicitly indicated in Eq. (3). In particular, the Mueller matrix describing the LD of the sample turns to be a function of a generic angle $\beta$, measured in the plane of the scan and describing, neglecting constant offsets, the orientation of the 
individual nanostructure under investigation. The spurious effect due to the optical fiber probe, described by $M_{i i}\left(\Delta \phi_{s p}\right)$, depends on a generic retardation $\Delta \phi_{s p}$. The time dependence of the radiation is clearly due to the periodical modulation of the retardation introduced by the photoelastic modulator, described by $M_{i}(t)$.

Demodulation at the first and the second harmonics of the signal is accounted for by expanding the function describing the output radiation in Fourier components. This enables computing the intensity associated with the first and the second Fourier coefficients, $I_{1}$ and $I_{2}$, respectively, which, neglecting conversion factors, represent the Ist and IInd signals acquired in our experiment.

The dependence of $I_{1}$ and $I_{2}$ on the parameters entering Eq. (3) is complex. For instance, for non-negligible values of $\Delta \phi_{s p}$ the model predicts a non-monotonic behavior of $I_{2}$ as a function of $\beta$. However, assuming $\Delta \phi_{s p} \sim 0$, one gets

$$
\begin{gathered}
I_{1} \approx\left|I_{0} J_{1}(A) \kappa \sinh \left(\alpha_{C D}\right)\right| \\
I_{2} \approx\left|I_{0} J_{2}(A) \kappa \sinh \left(\alpha_{L D}\right) \cosh \left(\alpha_{C D}\right) \cos (2 \beta)\right|
\end{gathered}
$$

where $\kappa$ is proportional to the optical thickness (isotropic absorption) of the material, as can be assessed from the DC signal maps, $J_{l}$ and $J_{2}$ are the first and second order canonical Bessel's functions, respectively $\left[J_{l}=0.52\right.$ and $J_{2}=0.43$, for the used maximum PEM retardation, $A=2.405]$, and $\alpha_{C D}$ and $\alpha_{L D}$ are the already mentioned coefficients ruling CD and LD. They can in turn be written as a function of the extinction coefficients for left- and right-handed circular polarization, $\varepsilon_{-}$and $\varepsilon_{+}$, and for linear polarization along two mutually orthogonal directions, $\varepsilon_{p a r}$ and $\varepsilon_{o r t}$, by

$$
\begin{gathered}
\alpha_{C D}=\frac{2 \pi}{\lambda}\left(\varepsilon_{-}-\varepsilon_{+}\right) z \\
\alpha_{L D}=\frac{2 \pi}{\lambda}\left(\varepsilon_{p a r}-\varepsilon_{o r t}\right) z
\end{gathered}
$$

where $\lambda$ is the laser wavelength and $z$ is the local thickness of the material, as can be assessed from the topography maps.

Equations $(4,5)$ can be further approximated in the case of thin and weakly absorbing samples, with $\alpha_{C D}$ and $\alpha_{L D}$ smaller than the unity, as expected in our case, leading to

$$
\begin{gathered}
I_{1} \approx\left|I_{0} J_{1}(A) \kappa \alpha_{C D}\right| \\
I_{2} \approx\left|I_{0} J_{2}(A) \kappa \alpha_{L D} \cos (2 \beta)\right| .
\end{gathered}
$$

Therefore, it is clear that, in such conditions, the coefficients ruling CD and LD can be independently and simultaneously deduced from the Ist and IInd signal maps acquired in our scans. In agreement with the experimental findings, Eqs. $(9,10)$ confirm that no dependence on the angular variable $\beta$, representative of the nanostructure orientation relative to the substrate, is expected for $I_{1}$, whereas a $\cos (2 \beta)$ dependence is foreseen for $I_{2}$. Such a specific behavior can be obtained only assuming negligible birefringence stemming from the optical fiber probe. In other words, $I_{1}$ turns independent of $\beta$ only if the conditions leading to Eqs. $(9,10)$ are satisfied. As a consequence, the occurrence of negligible spurious contributions from specific optical fiber probes can be assessed a posteriori, by analyzing the eventual dependence of the Ist signal, measured over individual nanostructures, on their angular orientation.

We have carried out a comprehensive investigation by scanning different portions of the sample and we have identified several tens of ribbon-shaped nanoaggregates, with typical width $0.2-0.5 \mu \mathrm{m}$, length $0.6-2.0 \mu \mathrm{m}$, and thickness $20-30$ $\mathrm{nm}$. For every identified nanoaggregate, the angular orientation referred to an arbitrary reference direction, hence corresponding to $\beta$, has been determined from the topographical maps and the corresponding values of Ist and IInd signals have been evaluated by spatial averaging the relevant optical maps on the whole nanostructure size. In order to get rid of unavoidable fluctuations in the laser intensity coupled to the optical fiber probe, resulting in scan-to-scan variations of the intensity $I_{0}$ entering Eqs. $(9,10)$, the measured Ist and IInd signals have been normalized to a reference value derived from the corresponding DC maps.

Figure 5 summarizes the results, showing the so-computed Ist and IInd signals, in arbitrary units normalized to unity, as a function of $\beta$. A conventional uncertainty is attributed to the computed values in order to account for signal fluctuations 
and for the error stemming from spatial averaging. Uncertainty in the evaluation of $\beta$ is mostly due to the non-perfectly linear shape of the identified nanoaggregates.

As expected, the Ist signal, representative of $\mathrm{CD}$, is practically constant, within the error bars, for all the observed nanoaggregates, whereas the IInd signal follows a marked trend, reasonably well reproduced by the $\cos (2 \beta)$ function superposed to experimental data. Furthermore, by determining the actual conversion factors relating the output of the lock-in amplifiers to the laser intensity $I_{0}$ and taking into account the effective optical thickness $\kappa$ of the observed nanoaggregates, as assessed from the DC maps, Eq. (9) leads to $\alpha_{C D} \sim 5 \times 10^{-3}$, i.e., the same order of magnitude of the corresponding coefficient found in CD spectroscopy of similar nanoaggregates in solution ${ }^{4}$.

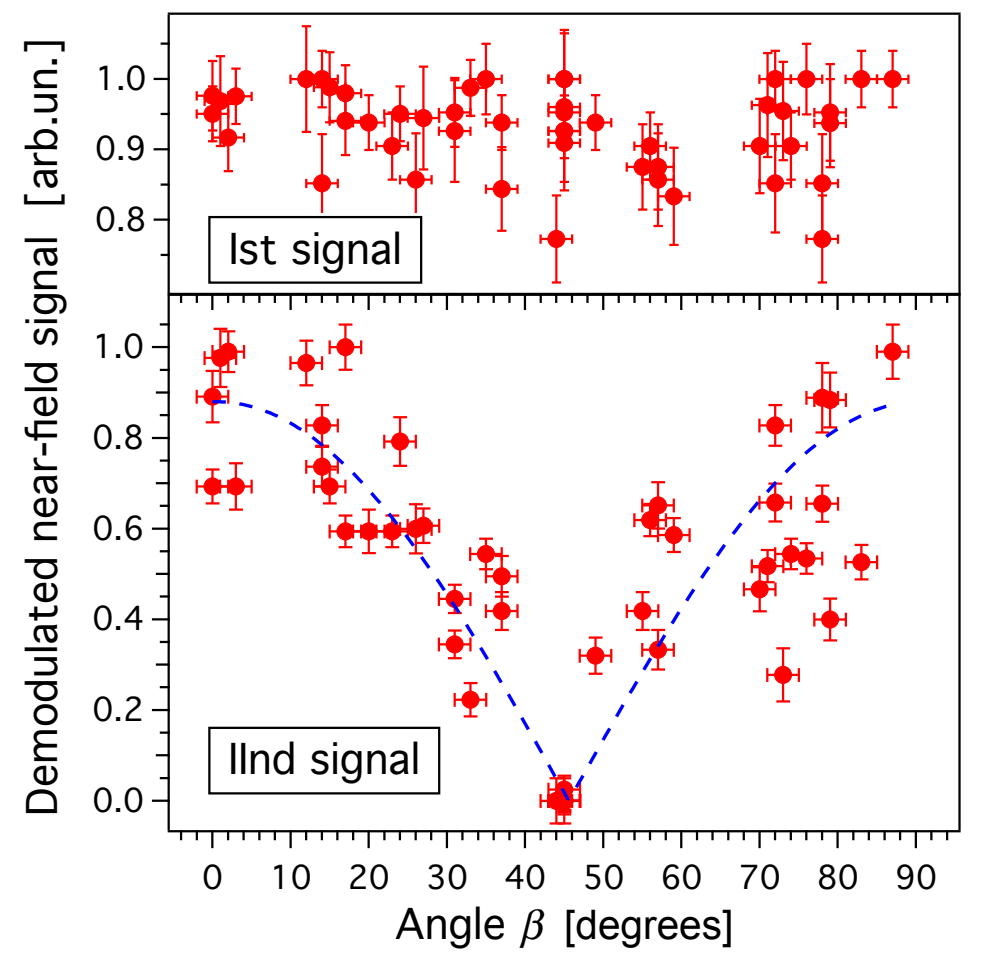

Figure 5. Near-field optical signal demodulated at the first and the second harmonics (Ist and IInd, respectively) as a function of the angle $\beta$ describing the orientation of the identified ribbon-shaped TPPS ${ }_{3}$ nanoaggregates in the scan plane. Values have been determined as discussed in the text. Note the different vertical scales for the two panels.

\section{CONCLUSIONS}

The application of a specifically conceived PM-SNOM setup to ribbon-shaped porphyrin nanoaggregates anchored onto a glass substrate leads to a reliable analysis of the optical activity of such nanostructures. In particular, demodulation at the first and the second harmonics of the near-field optical signal acquired in a configuration mimicking conventional transmission microscopy produces maps representative of CD and LD, respectively.

A model of the setup based on the Mueller matrix formalism confirms the possibility to measure, simultaneously and independently, the response to linear and circular polarized light. The model suggests as well a method, which can be verified a posteriori, to assess the absence of relevant spurious effects due to the residual birefringence of the optical fiber probe, which could entangle LD and CD. The substantial independence of the Ist signal on the angle $\beta$ displayed in Fig. 5 and the $\cos (2 \beta)$ dependence of the simultaneously acquired IInd signal well demonstrates the statement above.

In other words, the proposed method is able to produce reliable, and even quantitative, measurements of LD and CD coefficients at the solid-state, while keeping the sub-diffraction spatial resolution typical of optical near-field 
microscopy. Its application to the porphyrin nanoaggregates demonstrates the circular dichroism properties are inherent with their structure, being based on the molecular arrangement of such J-aggregates, whereas the linear dichroism can be ascribed to the elongated and anisotropic shape, leading to a marked dependence of the observed signals on the nanoaggregate orientation.

\section{REFERENCES}

[1] Amabilino, D. B., [Chirality at the nanoscale: nanoparticles, surfaces, materials and more], Wiley-VCH, Weinheim, (2009).

[2] Cotton, A., "Recherches sur l'absorption et la dispersion de la lumière par les milieux doués du pouvoir rotatoire", J. Phys. Theor. Appl., 5(1) 237-244 (1896).

[3] Gucciardi, P. G., Micheletto, R., Kawakami, Y. and Allegrini, M., "Polarization-modulation techniques in nearfield optical microscopy for imaging of polarization anisotropy in photonic nanostructures", Chapter 10 in [Applied Scanning Probe Methods II], Bhushan, B. and Fuchs, H., Editors, Springer, Berlin Heidelberg (2006).

[4] Tantussi, F., Fuso, F., Allegrini, M., Micali, N., Occhiuto, I. G., Monsù Scolaro, L. and Patanè, S., "Linear and circular dichroism in porphyrin J-aggregates probed by polarization modulated scanning near-field optical microscopy", Nanoscale, 6, 10874-10878 (2015).

[5] Xyaoyang, D., Song, Y. and Na, L., "Understanding complex chiral plasmonics", Nanoscale, 7, 17237-17243 (2015).

[6] Xinghui, Y., Schaeferling, M., Michel, A.-K- U., Tittl, A. Wuttig, M., Taubner, T. and Giessen, H., “Active chiral plasmonics", Nano Lett., 15(7), 4255-4260 (2015).

[7] Gucciardi, P. G., Labardi, M., Gennai, S., Lazzeri, F. and Allegrini, M., "Versatile scanning near-field optical microscope for material science applications”, Rev. Sci. Instrum., 68, 3088-3093 (1997).

[8] Micali, N., Engelkamp, H., van Rhee, P. G., Christiansen, P. C. M., Monsù Scolaro, L. and Maan, J. C., "Selection of supramolecular chirality by application of rotational and magnetic forces", Nat. Chem., 4, 201207 (2012).

[9] Rubires, R., Farrera, J. A. and Ribó, J. M., "Stirring effects on the spontaneous formation of chirality in the homoassociation of diprotonated meso-tetraphenylsulfonato porphyrins", Chem.-Eur. J., 7, 436-446 (2001).

[10] Escudero, C., Crusats, J., Díez-Pérez, I., El-Hachemi, Z. and Ribò, J. M., "Folding and hydrodynamic forces in J-aggregates of 5-phenyl-10, 15, 20-tris (4-sulfophenyl) porphyrin", Angew. Chem. Int. Ed., 118(47), 82008203 (2006).

[11] Ramoino, L., Labardi, M., Maghelli, N., Pardi, L. and Allegrini, M., "Polarization-modulation near-field optical microscope for local dichroism mapping”, Rev. Sci. Instrum., 73, 2051-2056 (2002).

[12] Ambrosio, A., Alderighi, M., Labardi, L., Pardi, L., Fuso, F., Nannizzi, S., Pucci, A. and Ruggeri, G., "Nearfield optical microscopy of polymer-based films with dispersed terthiophene chromophores for polarizer applications", Nanotechnology, 15(4), 270-275 (2004).

[13] Tantussi, F., Menghetti, S., Caldi, E., Fuso, F., Allegrini, M. and Galli, G., "Pure optical and reversible optically driven nanowriting of azobenzene block copolymers", Appl. Phys. Lett., 100, 083103 (2012).

[14] Camposeo, A., Greenfeld, I., Tantussi, F., Pagliara, S., Moffa, M., Fuso, F., Allegrini, M., Zussman, E. and Pisignano, D., "Local mechanical properties of electrospun fibers correlate to their internal structure", Nano Lett., 13(11), 5056-5062 (2013).

[15] Camposeo, A., Greenfeld, I., Tantussi, F., Moffa, M., Fuso, F., Allegrini, M., Zussman, E. and Pisignano, D., "Conformational evolution of elongated polymer solutions tailors the polarization of light-emission from organic nanofibers", Macromol., 47(14), 4704-4710 (2014).

[16] Hecht, E. and Zajac, A., [Optics Third Edition], Addison Wesley, Boston (1998). 